\title{
10-40GHz design in SiGe-BiCMOS and Si-CMOS - linking technology and circuits to maximize performance
}

\author{
H. Veenstra ${ }^{1}$, G.A.M. Hurkx ${ }^{1}$, E. v.d. Heijden ${ }^{1}$, C.S. Vaucher ${ }^{1}$, M. Apostolidou ${ }^{1}$, D. Jeurissen ${ }^{1}$, \\ P. Deixler ${ }^{2}$ \\ ${ }^{1}$ Philips Research Laboratories, Prof. Holstlaan 4, 5656 AA Eindhoven, The Netherlands, 31402743606 \\ ${ }^{2}$ Philips Semiconductors, 2070 Route 52, P.O. Box 1279, Hopewell Junction, NY 12533, USA
}

\begin{abstract}
This paper reviews the relevance of the widely used device metrics $f_{T}, f_{\max }$ as well as the recently introduced device metrics $f_{A}$ and $f_{\text {cross }}$ for broadband circuit design. Usually, IC processes are benchmarked on the basis of their $f_{T}$ and $f_{\text {max }}$. For most circuit applications however, there is only an indirect relation between $f_{T}, f_{\max }$ and circuit bandwidths. Since the differential pair amplifier is a key building block in broadband circuits, the metric $f_{A}$ provides a nearly direct relation to broadband circuit performance. This is demonstrated via the maximum operating frequency of a current-mode logic frequency divider, processed in 3 generations of a BiCMOS process. Metric $f_{\text {cross }}$ is valuable for the design of circuits employing a cross-coupled differential pair as active negative resistance, such as in LCVCOs. The metrics can be expressed in terms of transistor parameters (e.g., $\left.\mathbf{R}_{b}, C_{b c}, \ldots\right)$, allowing to derive a link between circuit performance and technology. Based on our experience, we evaluate IC processes on the basis of $f_{T}, f_{A}$ and $f_{\text {cross }}$ rather than $f_{T}$ and $f_{\text {max }}$.
\end{abstract}

\section{INTRODUCTION}

It is common practice to use the transition frequency $f_{T}$ and maximum oscillation frequency $f_{\max }$ for benchmarking and IC process optimization. However, since there is only an indirect relation between the device metrics $f_{T}, f_{\max }$ and broadband circuit performance, optimizing a process on the basis of these metrics does not automatically lead to optimum circuit bandwidths. Thus, there is a need for device metrics that provide on the one hand a direct relation to broadband circuit bandwidths and, on the other hand, provide a direct relation to device parameters (i.e., $\mathrm{R}_{\mathrm{b}}, \mathrm{C}_{\mathrm{bc}}, \ldots$ ). In this paper, the relevance of two recently introduced device metrics for broadband circuit design is analyzed, namely the available bandwidth $f_{A}$ [1] and the maximum frequency for which a cross-coupled differential pair provides a negative shunt input resistance $f_{\text {cross }}$ [2].

In Section II, the device metric definitions are reviewed for bipolar transistors, and their relation to transistor parameters of a simplified transistor model is provided. In Section III, the evolution of these metrics across 3 generations of a BiCMOS process is reviewed. While the transition from [3] to [4] was driven by metrics $f_{T}$ and $f_{\max }$, the transition from [4] to [5] was driven by $f_{T}$, $f_{A}$ and $f_{\text {cross. }}$. Despite the increase in $f_{T}$ and $f_{\text {max }}$ from [3] to [4], a relatively poor improvement in broadband circuit bandwidths was found. Reversely, the transition from [4] to [5] resulted in a significant circuit performance increase. In Section IV, some circuit examples and their relation to device metrics will be described. Section $\mathrm{V}$ highlights the major differences between bipolar and CMOS for $f_{A}$ and $f_{\text {cross }}$, as well as the implications for broadband circuit design. The paper ends with conclusions in Section VI.

\section{DEVICE METRICS FOR BIPOLAR}

Although device metrics can be derived directly from measured y-parameters [1], in this paper all metrics are obtained from Spectre circuit simulations. To obtain simple relations between the metrics and transistor parameters, they are evaluated for the simplified equivalent transistor circuit shown in Fig. 1. In all circuits in this section, dc biasing has been omitted.

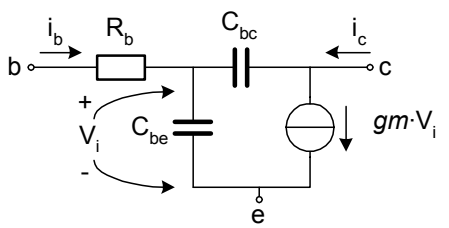

Fig. 1: Equivalent small-signal circuit diagram.

$$
\text { A. } f_{T} \text { and } f_{\text {max }}
$$

For the derivation of $f_{T}$, the base is driven by a current source while the collector is grounded. For the derivation of $f_{\max }$, the base is driven by a port with source impedance $Z_{s}$ and terminated by a load resistance $Z_{L}$, each optimized for power matching. The corresponding circuits are shown in Fig. 2. (a)

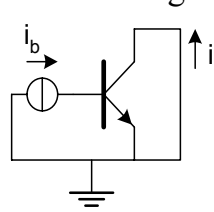



(b)
Fig. 2: Schematic to extract $f_{T}$ (a) and $f_{\max }$ (b).

Both configurations are not representative for broadband circuits. The base terminal is usually driven via a low-ohmic source impedance. Metric $f_{T}$ represents the bandwidth of the current gain of a common-base amplifier. Metric $f_{\max }$ has no direct relation to broadband circuit performance, since wideband operation is typically obtained by matching to the interconnect characteristic impedance, and transistor in- and output impedance are considered as parasitics. 
A further drawback of metrics $f_{T}$ and $f_{\max }$ is that the peak-values of modern bipolar and CMOS processes are well beyond the measurement capabilities of even the most advanced equipment. Thus, the results rely on extrapolation. Besides, metric $f_{\max }$ can be based either on the maximum stable gain or the unilateral gain, which complicates the relation to broadband circuit bandwidths.

$$
\text { B. } f_{A}, f_{V} \text { and } f_{\text {out }}
$$

The available bandwidth $f_{A}$ represents the 3-dB bandwidth of a differential pair amplifier driven by a voltage source, designed for $20 \mathrm{~dB}$ low-frequency voltage gain. Across a bias sweep, the load resistance value $\mathrm{R}_{\mathrm{L}}$ is adjusted to fix the low-frequency voltage gain to $20 \mathrm{~dB}$. Metric $f_{A}$ can be sub-divided in two contributions, namely the input bandwidth $f_{V}$ (representing the bandwidth of the transconductance $\left.i_{c} / v_{b e}\right)$ and output bandwidth $f_{\text {out }}$. The single-ended equivalent circuits for the derivation of $f_{V}, f_{\text {out }}$ and $f_{A}$ are shown in Fig. 3.

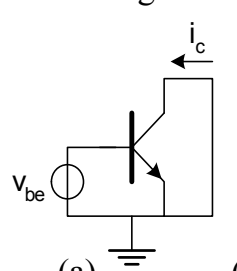

(a)

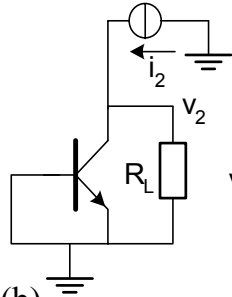

(b)

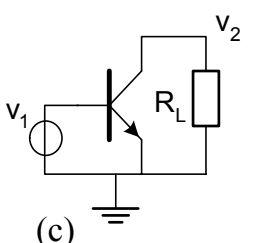

(c)
Fig. 3: Schematic to extract $f_{V}(\mathrm{a}), f_{\text {out }}$ (b) and $f_{A}$ (c).

For the equivalent transistor circuit shown in Fig. 1, the following approximations can be derived:

$$
\begin{gathered}
f_{V}=\frac{1}{2 \pi R_{b}\left(C_{b e}+C_{b c}\right)}=\frac{f_{T}}{g m \cdot R_{b}} \\
f_{\text {out }}=\frac{1}{2 \pi R_{L} C_{b c}\left(1+g m \cdot R_{b}\right)}=\frac{1}{2 \pi R_{L} C_{22}} \\
f_{A}=\frac{f_{V} f_{\text {out }}}{f_{V}+f_{\text {out }}}
\end{gathered}
$$

Here, $\mathrm{C}_{22}=\mathrm{C}_{\mathrm{bc}}\left(1+g m \cdot \mathrm{R}_{\mathrm{b}}\right)$ is the transistor output capacitance. When evaluating $f_{A}$ across a bias sweep, the following observations can be made. In the first place, metric $f_{A}$ is dominated by $f_{\text {out }}$ for low currents (where high load resistance values are required due to the low transconductance $g m$ ) and by $f_{V}$ for high currents (where the base-emitter capacitance $\mathrm{C}_{\mathrm{be}}$ is high). The output capacitance $\mathrm{C}_{22}$ is constant at low bias currents, but is proportional to the bias current when $g m \cdot \mathrm{R}_{\mathrm{b}}>1$. This effect is demonstrated in Fig. 4. As a consequence, the output bandwidth saturates for $\mathrm{I}_{\mathrm{c}}>\mathrm{V}_{\mathrm{T}} / \mathrm{R}_{\mathrm{b}}$, with $\mathrm{V}_{\mathrm{T}} \approx 25 \mathrm{mV}$ the thermal voltage. Since the input bandwidth $f_{V}$ decreases with increasing bias current, the peak- $f_{A}$ typically occurs at a bias current where $g m \cdot \mathrm{R}_{\mathrm{b}} \approx 1$ to 2 .

\section{C. $f_{\text {cross }}$}

Metric $f_{\text {cross }}$ represents the highest frequency for which a cross-coupled differential pair provides a negative shunt input resistance. At $f_{\text {cross }}$, the real part of the input admittance crosses zero. The equivalent circuit for the derivation of $f_{\text {cross }}$ is shown in Fig. 5. A virtual ground exists at the common emitter node, where a dc bias current is applied.



Fig. 4: Effect of the base series resistance $R_{b}$ on the output capacitance $\mathrm{C}_{22}$ and bandwidth $f_{\text {out }}$.

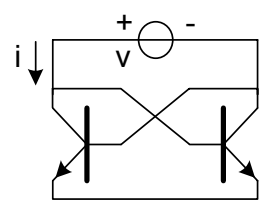

Fig. 5: Circuit to extract $f_{\text {cross }}$.

For the circuit of Fig. 1, $f_{\text {cross }}$ equals

$$
f_{\text {cross }} \approx f_{T} \sqrt{\frac{1}{g m \cdot R_{b}}}=\sqrt{f_{V} \cdot f_{T}}
$$

To obtain a high $f_{\text {cross }}$, a low base series resistance is essential.

For the SiGe process of [5], the device metrics across a bias sweep are shown in Fig. 6. The curves are valid for a $0.5 \times 4.7 \mu \mathrm{m}^{2} \mathrm{npn}$ transistor biased at $\mathrm{V}_{\mathrm{cb}}=0 \mathrm{~V}$.

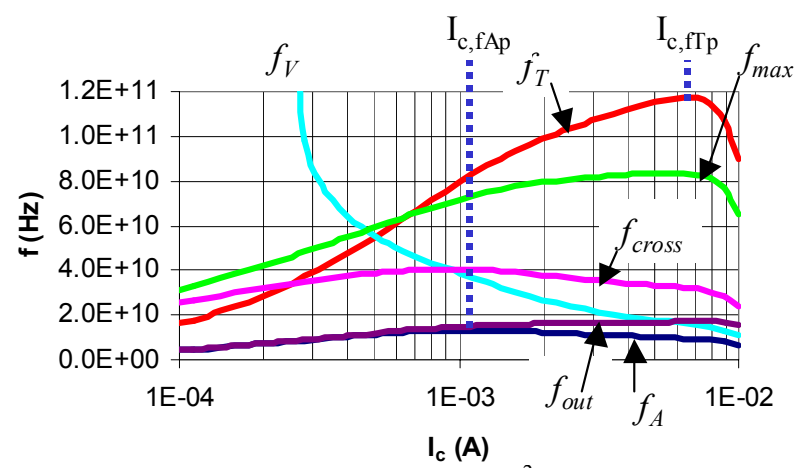

Fig. 6: Device metrics for a $0.5 \times 4.7 \mu \mathrm{m}^{2} \mathrm{npn}$ from [5].

From the curves shown in Fig. 6, some interesting observations can be made. Firstly, it can be seen that $f_{\text {cross }}$ is relatively independent of the bias current for an order of magnitude in bias current variations. This can be explained from equation (4). For bias conditions below the current density for peak- $f_{T}$, the increase in $f_{T}$ for increased bias current is compensated by a reduction in $f_{V}$. Secondly, from the crossing of the $f_{V}$ and $f_{T}$ curves follows that the condition $g m \cdot \mathrm{R}_{\mathrm{b}}=1$ occurs at a current density well before peak- $f_{T}$. Thirdly, the current density for peak $-f_{A}$ lays a factor of 5 below the current density for peak- $f_{T}$. This is due to the saturation of $f_{\text {out }}$ for currents where $g m \cdot \mathrm{R}_{\mathrm{b}}$ becomes larger than unity. At peak $-f_{A}$, the $f_{A}$ is dominated by the output bandwidth $f_{\text {out }}$. Such information is important for circuit design. A differential pair amplifier biased at peak- $f_{T}$ may not provide the maximum bandwidth in this process. 


\section{EVOLUTION OF THE DEVICE METRICS}

In Table I, the evolution of the device metrics across 3 generations of a $0.25 \mu \mathrm{m}$ BiCMOS process are shown for $\mathrm{V}_{\mathrm{cb}}=0 \mathrm{~V}$. The table refers to values obtained from Spectre circuit simulations. The last column is extracted from the $f_{T} / f_{V}$-ratio at peak- $f_{T}$, which provides a good approximation for $g m \cdot \mathrm{R}_{\mathrm{b}}$ as follows from equation (1).

\begin{tabular}{|l|l|l|l|l|l|l|}
\hline Process & Year & $\begin{array}{c}f_{T} \\
\mathrm{GHz}\end{array}$ & $\begin{array}{c}f_{\max } \\
\mathrm{GHz}\end{array}$ & $\begin{array}{c}f_{A} \\
\mathrm{GHz}\end{array}$ & $\begin{array}{l}f_{\text {cross }} \\
\mathrm{GHz}\end{array}$ & $\begin{array}{l}g m \cdot \mathrm{R}_{\mathrm{b}} \\
\mathrm{at} \mathrm{pk} f_{T}\end{array}$ \\
\hline$[3]$ & 2001 & 33 & 60 & 14.6 & 27 & 1.7 \\
\hline$[4]$ & 2002 & 61 & 73 & 15.2 & 34 & 2.3 \\
\hline$[5] \mathrm{a}$ & 2004 & 117 & 84 & 13.0 & 40 & 5.9 \\
\hline$[5] \mathrm{b}$ & 2004 & 109 & 90 & 15.9 & 47 & 5.0 \\
\hline$[5] \mathrm{c}$ & 2005 & 108 & 117 & 24 & 62 & 2.8 \\
\hline
\end{tabular}

Table I: Evolution of device metrics (peak values).

The transition from [3] to [4] was driven by an increase in peak- $f_{T}$, made possible through the introduction of a SiGe layer. The higher peak- $f_{T}$ is obtained at an increased current density. The base series resistance $R_{b}$ remained constant. This resulted in an increased $g m \cdot \mathrm{Rb}$ at peak- $f_{T}$, and thus a more dominant effect of the output bandwidth to $f_{A}$ due to the Miller effect in $C_{b c}$ as follows from equation (2). Process [5] is currently still under development. In Table I the processes [5]a, [5]b and [5]c represent three stages in the process development. In the initial stage [5] a the maximum $f_{T}$ was increased, mainly by the introduction of carbon into the base, which inhibits boron diffusion. The accompanied increase in base- and collector doping resulted in an increased base-collector capacitance $\mathrm{C}_{\mathrm{bc}}$. In combination with an approximately constant $R_{b}$ (dominated by the extrinsic part), this resulted in a reduction of the output bandwidth $f_{\text {out }}$, explaining the reduced $f_{A}$ in [5]a, despite the increased $f_{T}$ and $f_{\max }$. In a second step ([5]b), process spreading was reduced, which allows a narrower emitter and consequently a higher $f_{\max }$ and $f_{A}$. In a third step ([5]c), the base resistance was further reduced by about a factor of two.

For process variants [3], [4] and [5]a, the peak- $f_{A}$ occurs at an approximately constant current density (where $g m \cdot \mathrm{R}_{\mathrm{b}} \approx 1$ to 2 ), and thus the ratio between the current densities for peak- $f_{T}$ versus peak- $f_{A}$ increased when migrating from [3] to [5]a. This is an undesired effect, since differential pair amplifiers operated at peak$f_{T}$ have a reduced bandwidth in the newer generation, despite the increase in $f_{T}$ and $f_{\max }$. The reduction of $\mathrm{R}_{\mathrm{b}}$ by a factor of two in [5]c shifts the saturation of $f_{\text {out }}$ to higher currents resulting in a substantially increased peak- $f_{A}$ and $f_{\text {cross. }}$. In a final step, $f_{T}$ and $f_{\max }$ were further increased to values around $130 \mathrm{GHz}$ without compromising $f_{A}$ and $f_{\text {cross }}$. For these results, no parameters are available yet and therefore this process step has not been included in Table I. The increase in $f_{A}$ and $f_{\text {cross }}$ is reflected in broadband circuit performance, as demonstrated in the next section.

\section{CIRCUIT EXAMPLES}

Metric $f_{A}$ is defined for a low-frequency gain of $20 \mathrm{~dB}$. Such a high gain is not widely used, and puts extra emphasis on the output bandwidth of a transistor. For example, bipolar current-mode logic (CML) circuits typically operate at a small-signal gain of 6 to $12 \mathrm{~dB}$. The extra emphasis on the output bandwidth accounts for loading effects, which is necessary since in the definition of $f_{A}$, the output is unloaded. Note that circuit bandwidths may also exceed $f_{A}$.

Circuit simulations and measurements revealed only a minor increase in the maximum operation frequency of a static CML frequency divider, from $20 \mathrm{GHz}$ in [3] (see [6]) to about $25 \mathrm{GHz}$ in [4] (see [7]). The circuit was recently processed in [5]c, and operated up to $40 \mathrm{GHz}$. The maximum operating frequency of the divider is proportional to the peak- $f_{A}$. All 3 circuits support input frequencies up to approximately $1.5 \cdot f_{A}$. The same conclusion was found during the design of a crossconnect switch for optical networking applications [8]. The design was implemented in [4] since this process features two thick top interconnect layers, needed for low-loss transmission lines for rows and columns in the cross-connect matrix. In every signal path through the matrix, about 10 differential pair amplifiers are cascaded. The circuit supports input data rates up to $f_{A}$.

The next circuit example deals about a pseudo-random binary sequence (PRBS) generator in an InP HBT process [9]. This process has a peak- $f_{A}$ of approximately $25 \mathrm{GHz}$. Our PRBS generator implemented in this process [10], based on CML circuits, supports output bit rates up to about $2 \cdot f_{A}$. Due to the half-rate architecture, the majority of the circuits operate at $f_{A}$, except for the output multiplexer and buffer, which operate at $2 \cdot f_{A}$.

For LC-VCO design, metric $f_{\text {cross }}$ is valuable. An LCVCO can be implemented for output frequencies close to $f_{\text {cross }}$ [2]. A capacitively loaded emitter follower allows the implementation of a negative resistance that is feasible at frequencies beyond $f_{\text {cross }}$. Such a topology is also used in a Colpitts oscillator. This topology does not lead to different requirements to the transistor compared to the requirements for high $f_{\text {cross }}$.

\section{CMOS VERSUS BIPOLAR}

For CMOS, $f_{A}$ is dominated by $f_{\text {out }}$ since the transistor layout can be optimized for very low gate series resistance $\mathrm{R}_{\mathrm{g}}$ and thus high $f_{V}$, (e.g., $f_{V}>100 \mathrm{GHz}$ is often feasible). When comparing bipolar versus CMOS, a CMOS process with comparable $f_{T}$ and $f_{\max }$ may possess a relatively poor $f_{A}$, as shown in Table II, where a $0.12 \mu \mathrm{m}$ CMOS process is compared with the SiGe BiCMOS process from [4]. Despite the favorable $f_{T}$ and $f_{\text {max }}$, the $f_{A}$ is substantially lower in the CMOS process. This is due to the relatively low transconductance, requiring high load resistance values that reduce the output bandwidth.

\begin{tabular}{|c|c|c|c|c|}
\hline Process & $\begin{array}{c}f_{T} \\
(\mathrm{GHz})\end{array}$ & $\begin{array}{c}f_{\max } \\
(\mathrm{GHz})\end{array}$ & $\begin{array}{c}f_{A} \\
(\mathrm{GHz})\end{array}$ & $\begin{array}{c}f_{\text {cross }} \\
(\mathrm{GHz})\end{array}$ \\
\hline CMOS12 & 86 & 138 & 6.7 & 123 \\
\hline$[4]$ & 61 & 73 & 15.2 & 34 \\
\hline
\end{tabular}

Table II : Example CMOS metrics, compared with a bipolar process with somewhat lower $f_{T}$ and $f_{\max }$. 
Furthermore, the higher impedance level in CMOS technology makes the impact of interconnect parasitic capacitances more important, and thus it is more difficult to realize circuit bandwidths predicted by $f_{A}$.

For CMOS, the favorable $f_{V}$ also results in a favorable $f_{\text {cross. }}$. Thus, the realization of microwave LC-VCOs in CMOS is usually not a problem, even in relatively outdated process generations. This statement is supported by a $38.7-41.8 \mathrm{GHz}$ VCO that was implemented in 0.18 $\mu \mathrm{m}$ CMOS with $f_{\text {cross }}>100 \mathrm{GHz}$. The chip photomicrograph is shown in Fig. 7, the output spectrum at $40 \mathrm{GHz}$ shown in Fig. 8.

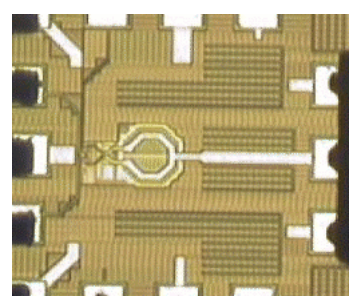

Fig. 7: $40 \mathrm{GHz} \mathrm{LC}-\mathrm{VCO}$ in $0.18 \mu \mathrm{m}$ CMOS.

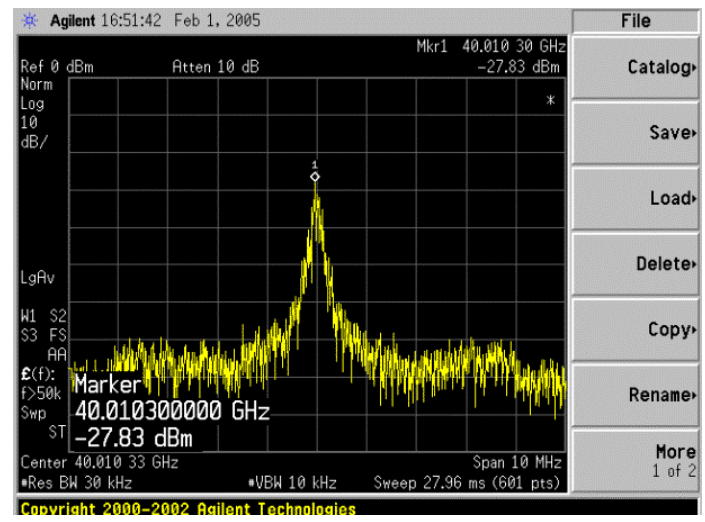

Fig. 8: Measured output spectrum at $40 \mathrm{GHz}$.

The relatively low oscillator output power is due to the $-23 \mathrm{~dB}$ voltage gain of differential pair output buffer, driving the external $50 \Omega$ load. This confirms that signal distribution and frequency division are more a challenge than signal generation in CMOS.

\section{CONCLUSION}

The available bandwidth $f_{A}$, representing the $3-\mathrm{dB}$ bandwidth of a differential pair amplifier designed for 20 $\mathrm{dB}$ low-frequency voltage gain, is a valuable performance indicator for broadband circuit applications. A technology with superior $f_{T}$ and $f_{\max }$ may have a lower $f_{A}$, and therefore not show the expected speed advantage. Metric $f_{A}$ can be subdivided into two contributions: the input bandwidth $f_{V}$ and output bandwidth $f_{\text {out }}$. Since IC processes are typically designed to allow current densities up to operation for peak- $f_{T}$, it is important to avoid saturation of $f_{\text {out }}$ up to such current densities. If this can be realized, the current density for peak- $f_{A}$ will be close to the current density for peak $f_{T}$. For a given technology, analyzing $f_{V}, f_{\text {out }}$ and $f_{T}$ across a bias sweep provides information about the main limiting factor to $f_{A}$, and shows whether the IC process is well balanced between in- and output bandwidths. The output bandwidth saturates for current levels above $g m \cdot \mathrm{R}_{\mathrm{b}}=1$ due to the Miller effect of $\mathrm{C}_{\mathrm{bc}}$. The bias point where $g m \cdot \mathrm{R}_{\mathrm{b}}=1$ corresponds to the crossing of the $f_{V}$ and $f_{T}$ curves. Broadband circuits are demonstrated that support data rates up to $f_{A}$ (for a complex cross-connect switch) up to $2 \cdot f_{A}$ (for CML frequency divider and PRBS generator).

A CMOS transistor can be designed for very low gate series resistance, thereby achieving an extremely high input bandwidth $f_{V}$. Thus, $f_{A}$ is dominated by the output bandwidth $f_{\text {out }}$. The high $f_{V}$ also results in a relatively high $f_{\text {cross }}$, even for mature CMOS processes. Thus, microwave VCOs can be implemented in mature CMOS technologies (as demonstrated), but the performance of CMOS processes with favorable $f_{T}$ and $f_{\max }$ for broadband circuit design does not yet reach the capabilities of BiCMOS due to a relatively poor $g m$ and $f_{A}$.

Based on our experience, we evaluate IC processes on the basis of $f_{T}, f_{A}$ and $f_{\text {cross }}$ rather than $f_{T}$ and $f_{\max }$.

\section{ACKNOWLEDGEMENT}

The authors wish to acknowledge Denis Crespo of Philips Semiconductors, Caen, France for his contribution to the QUBiC4X frequency divider.

\section{REFERENCES}

[1] G.A.M. Hurkx, P. Agarwal, R. Dekker, E. v.d. Heijden and H. Veenstra, "RF Figures-of-Merit for Process Optimization," IEEE Trans. Electron Devices, vol. 51, Dec. 2004, pp. 2121-2128.

[2] H. Veenstra, E. van der Heijden "A 19-23 GHz Integrated LC-VCO in a production $70 \mathrm{GHz} \mathrm{f}_{\mathrm{T}} \mathrm{SiGe}$ technology," in Proc. ESSCIRC, 2003, pp. 349-352.

[3] D. Szmyd, R. Brock et al, "QUBiC4: A Silicon-RF BiCMOS Technology for Wireless Communication ICs," in Proc. IEEE BCTM, 2001, pp. 60-63.

[4] P. Deixler, R. Colclaser et al, "QUBiC4G: A $\mathrm{f}_{\mathrm{T}} / \mathrm{f}_{\max }=70 / 100 \mathrm{GHz} 0.25 \mu \mathrm{m}$ Low Power SiGe-BiCMOS Production Technology with High Quality Passives for $12.5 \mathrm{~Gb} / \mathrm{s}$ Optical Networking and Emerging Wireless Applications up to 20GHz," in Proc. IEEE BCTM, 2002, pp. 201-204

[5] P. Deixler, A. Rodriguez et al, "QUBiC4X: An $\mathrm{f}_{\mathrm{T}} / \mathrm{f}_{\max }=130 / 140 \mathrm{GHz} \quad$ SiGe:C-BiCMOS Manufacturing Technology with Elite Passives for Emerging Microwave Applications," in Proc. IEEE BCTM, 2004, pp. 233-236.

[6] C.S. Vaucher, M. Apostolidou, "A Low-Power $20 \mathrm{GHz}$ Static Frequency Divider wit Programmable Input Sensitivity," 2002 IEEE RFIC Symposium, pp. 235-238.

[7] C.S. Vaucher et al, "Silicon-Germanium ICs for Satellite Microwave Front-ends," to appear in Proc. IEEE BCTM, 2005.

[8] H. Veenstra, P. Barré, E. v.d. Heijden, D. van Goor, N. Lecacheur, B. Fahs, G. Gloaguen, S. Clamagirand, O. Burg, "A 20-Input 20-Output $12.5 \mathrm{~Gb} / \mathrm{s} \mathrm{SiGe}$ Crosspoint Switch for Optical Networking with <2ps RMS jitter," ISSCC Dig. Tech. Papers, 2003, pp. 174-175.

[9] N.X. Nguyen, J. Fierro, G. Peng, A. Ly and C. Nguyen, "Manufacturable Commercial 4-inch InP HBT Device Technology," in Proc. GaAs MANTECH, 2002.

[10] H. Veenstra, "1-58 Gb/s PRBS generator with $<1.1$ ps RMS jitter in InP technology," in Proc. ESSCIRC, 2004, pp. 359-362.

[11] H. Veenstra, E. van der Heijden, "A 35.2-37.6 GHz LCVCO in a $70 / 100 \mathrm{GHz} f_{T} / f_{\max }$ SiGe technology," in Proc. ISSCC, 2004, pp. 359-362. 